\title{
Optimizing STEM Optics for EELS of Amorphous and Crystalline Materials in Semiconductors
}

Amish Shah and Yoselin Rangel

Intel Corporation, Corporate Quality Network, Hillsboro, Oregon, United States

The semiconductor industry uses a wide range of materials including metals, insulators, and semiconductors to meet the challenges of shrinking device sizes for logic devices. These materials can be single crystal, polycrystalline, and amorphous. The thickness of such layers such as high $\mathrm{K}$ dielectrics and spacers can approach less than a few nanometers, where the nanoscale properties of materials dominate the electrical behavior rather than bulk properties of materials. To understand material properties and potential failures of semiconducting chips, it is necessary to characterize both the structural properties such as crystallinity and strain, and chemical properties such as composition.

Often, materials can diffuse or oxidize through high temperature anneals or etches, leading to non-ideal compositions of neighboring materials. High spatial resolution EDX mapping can detect bulk diffusion of most materials down to 5-10\% composition. EELS can also be used at high spatial resolution to detect bulk diffusion of materials. In particular, light elements such as $\mathrm{Si}, \mathrm{C}$, and $\mathrm{O}$ have high collection efficiency of electron energy loss spectroscopy (EELS) signals. However, with a wide range of materials in semiconductor chips, there has not been an exhaustive study on optimizing convergence and collection angles to detect trace elements with EELS.

In this investigation, we collected EELS on amorphous, poly crystalline, and single crystal silicon to understand how to best optimize the EELS signal to background (StB). Blanket films of $\sim 200 \mathrm{~nm}$ of amorphous $\mathrm{Si}$ and polycrystalline Si were deposited on single crystal $\mathrm{Si}$ wafers with a SiOx separation layer between the film and substrate and cross sectioned with a FIB with final thinning at $2 \mathrm{kV}$. A probe corrected STEM was used to collect EELS with a number convergence and collection angles. The optimum convergence angle for maximum STEM resolution is $28 \mathrm{mrad}$.

EELS was collecting by scanning the beam over a $\sim 500 \times 100 \mathrm{~nm}$ area over the bulk films and the single crystal substrate with 21 and 28 mrad semiconvergence angles. 100-200 spectra were summed for each acquisition. For each camera length, we shifted the diffraction pattern to the center of the $2.5 \mathrm{~mm}$ entrance aperture, refocused the spectrometer to obtain a well-focused zero loss peak (ZLP), and shifted the ZLP center to $0 \mathrm{eV}$. When changing to the $5.0 \mathrm{~mm}$ entrance aperture, no refocusing of the spectrometer was performed, although it was noted that the FWHM of the ZLP did not change significantly. We collected the Si L and Si K edge for each sample with 10 semicollection angles ranging from $9 \mathrm{mrad}$ to $85 \mathrm{mrad}$. We measured the signal of the edge to the background as shown in Figure 1, with the signal of the background subtracted edge divided by the difference of the total signal and the background subtracted signal.

In general, choosing a collection angle close to the probe convergence angle maximizes the StB. For both edges and both convergence angles, it was found that a slightly smaller collection angle than convergence angle maximizes the StB. Surprisingly, amorphous Si needs an even smaller collection angle than poly crystalline or single or crystal $\mathrm{Si}$, indicating the forward inelastic scattering of a-Si differs from either of the crystalline polymorphs. It was also found that the smaller convergence angle of $21 \mathrm{mrad}$ resulted in $20 \%$ higher StB than the optimum angle of 28 mrad. For the Si K edge, the collection angle makes a dramatic difference in StB, but for the Si L edge, the collection angle has little impact. 


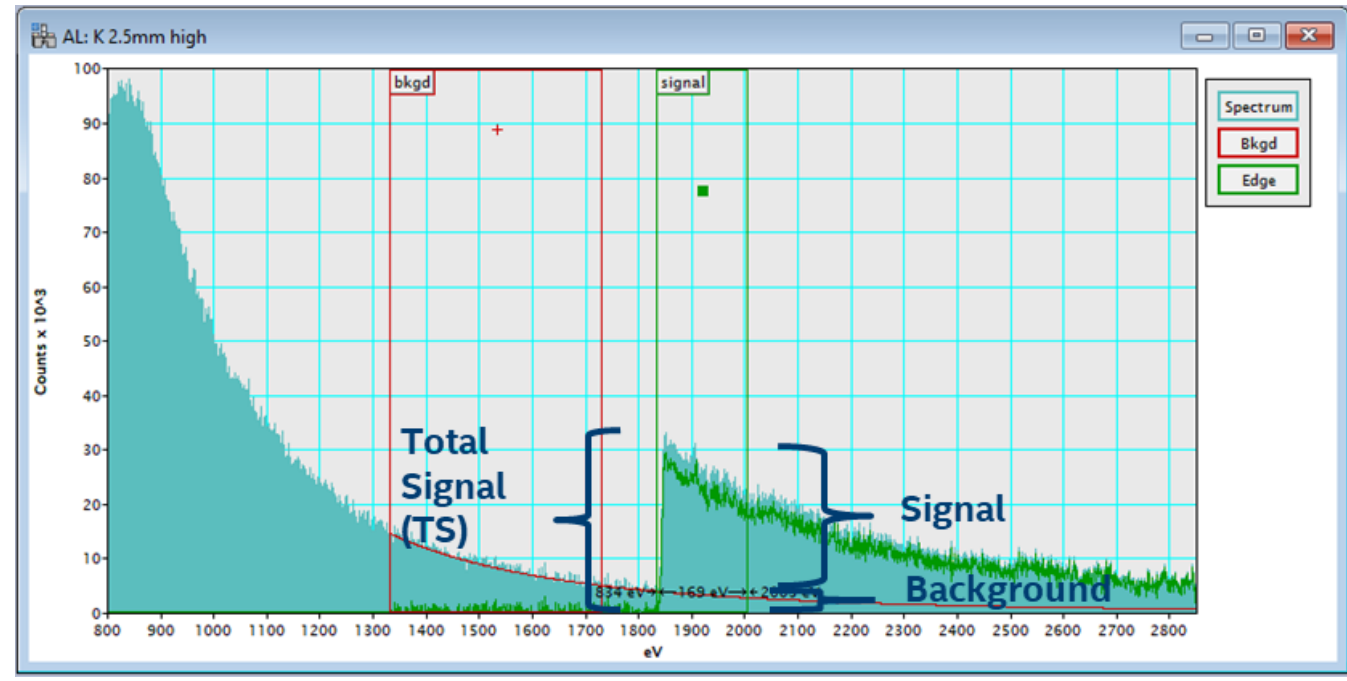

Figure 1. Figure 1: Method to measure signal to background (StB) in the Si K edge.

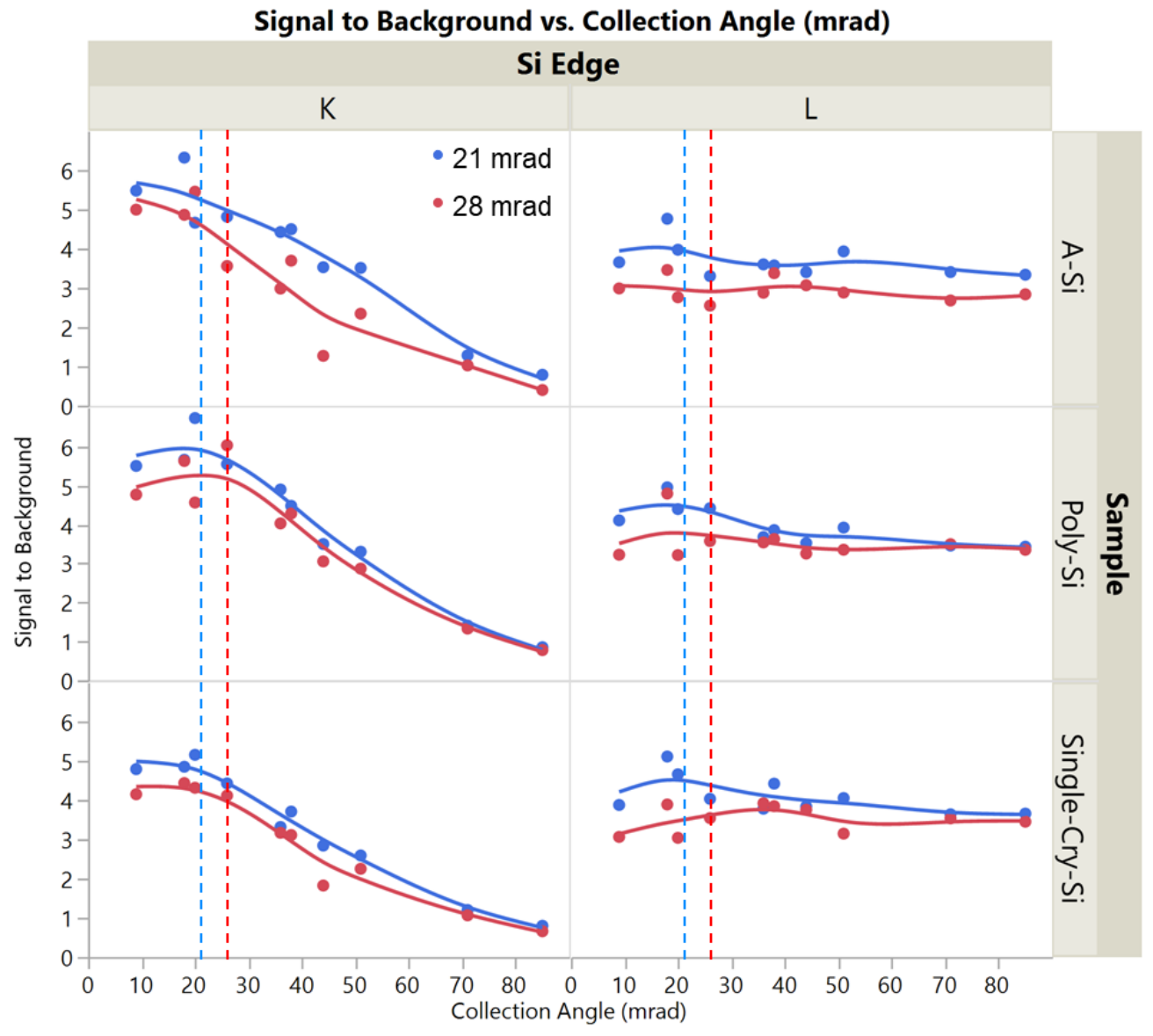

Figure 2. Figure 2: StB of Si K and Si L EELS edges of a-Si, poly-Si, and single crystal Si. The smoothed lines are guides to the eye. The vertical dashed lines indicate the probe convergence angle. The maximum $\mathrm{StB}$ is typically found with a collection angle a few mrad smaller than the probe convergence angle and with a smaller probe convergence angle of $21 \mathrm{mrad}$ than the optimum angle of 28 mrad for aberration corrected STEM. The Si K edge StB dramatically increases inversely with collection angle. a-Si has a smaller collection angle than poly-Si or single crystalline to maximize StB. 